\title{
A new sarcopenia score prognostic for postoperative complications in hepatic alveolar echinococcosis: a multicenter retrospective study
}

\author{
Tao Wang ${ }^{1 \#}$, Xianwei Yang ${ }^{1 \#}$, Wanxiang Wang ${ }^{2 \#}$, Tingyu Chen ${ }^{3}$, Junjie Kong ${ }^{1}$, Shu Shen ${ }^{1}$, Ying Chen ${ }^{4}$, \\ Gengfu Wei ${ }^{5}$, Dinggang Yu ${ }^{6}$, Cong Wang ${ }^{7}$, Minghao Li ${ }^{8}$, Shaozhen Rui ${ }^{9}$, Biao Luo ${ }^{10}$, Wentao Wang ${ }^{1}$ \\ ${ }^{1}$ Department of Liver Surgery and Liver Transplantation Center, West China Hospital of Sichuan University, Chengdu, China; ${ }^{2}$ Department of \\ Hepatobiliary, Pancreatic, and Splenic Surgery, The Affiliated Hospital of Inner Mongolia Medical University, Hohhot, China; ${ }^{3}$ Department of \\ Radiology, West China Hospital of Sichuan University, Chengdu, China; ${ }^{4}$ Department of Hepatobiliary Surgery I, The People’s Hospital of Ganzi \\ Tibetan Autonomous Prefecture, Kangding, China; ${ }^{5}$ Department of Hepatobiliary Surgery II, The People's Hospital of Ganzi Tibetan Autonomous \\ Prefecture, Kangding, China; 'Department of general Surgery, The People's Hospital of Aba Tibetan and Qiang Autonomous Prefecture, Barkam, \\ China; ${ }^{7}$ Department of Hepatopancreatobiliary surgery, the Affiliated Hospital of Qinghai University and Qinghai Province Key Laboratory of \\ Hydatid Disease Research, Qinghai University, Xining, China; ${ }^{8}$ Department of Hepatobiliary Surgery, The General Hospital of Ningxia Medical \\ University, Yinchuan, China; ${ }^{9}$ Department of General Surgery, The First Hospital of Lanzhou University, Lanzhou, China; ${ }^{10}$ Department of General \\ Surgery, Hospital of Chengdu Office, People's Government of Tibet Autonomous Region, China \\ Contributions: (I) Conception and design: T Wang, W Wang, W Wang; (II) Administrative support: W Wang, W Wang; (III) Provision of study \\ materials or patients: T Wang, X Yang, T Chen, J Kong, S Shen, Y Chen, G Wei, D Yu, C Wang, M Li, S Rui, B Luo; (IV) Collection and assembly \\ of data: T Wang, X Yang, T Chen, J Kong, S Shen, Y Chen, G Wei, D Yu, C Wang, M Li, S Rui, B Luo; (V) Data analysis and interpretation: T \\ Wang, X Yang, W Wang; (VI) Manuscript writing: All authors; (VII) Final approval of manuscript: All authors. \\ \#These authors contributed equally to this work. \\ Correspondence to: Wentao Wang. Department of Liver Surgery and Liver Transplantation Center, West China Hospital of Sichuan University, 37 \\ Guoxue Road, Chengdu 610041, China. Email: wwtdoctor02@163.com.
}

Background: Skeletal muscle depletion and excessive visceral adipose tissue have been shown to be independent risk factors for postoperative complications (PCs) in various diseases. However, their impact on surgical PCs in hepatic alveolar echinococcosis (HAE) is still unknown.

Methods: We retrospectively reviewed the clinical data of HAE patients who underwent liver resection at our hospital between January 2008 and December 2018. We segmented skeletal muscle and adipose tissue and measured the area of skeletal muscle tissue and adipose tissue at the level of the third lumbar vertebra by manual tracing from preoperative plain computed tomography (CT) images. Sarcopenia features were selected to construct a formula based on the least absolute shrinkage and selection operator (LASSO) logistic regression model in the primary set. Then, integrating the results of multiple clinicopathologic characteristics, we built a nomogram for predicting major PCs in HAE. The results were validated using bootstrap resampling and clinical data from other HAE centers in western China.

Results: The sarcopenia score is based on the personalized levels of the five features from the primary set $(n=233)$. In the multivariate logistic analysis of the primary set, the independent factors for PCs were $\gamma$-glutamyl transferase (GGT), and surface area of hepatectomy, which were integrated into the nomogram combined with sarcopenia score. The model had a good prediction capability with a C-index of 0.84 (95\% CI, 0.72-0.96). The calibration plot for the probability of PCs showed an optimal agreement between the nomogram predictions and actual observations in the primary and validation sets.

Conclusion: Our study showed that sarcopenia score was significantly correlated with PCs in patients with HAE. In addition, we constructed a prognostic nomogram for predicting complications in HAE patients after liver surgery. The nomogram displayed excellent discrimination and calibration. Improving the nutritional status and physical health of patients before surgery might reduce the incidence of postoperative complications for the high-risk patients. 
Keywords: Hepatic alveolar echinococcosis (HAE); postoperative complications; sarcopenia; visceral adiposity; least absolute shrinkage and selection operator (LASSO) logistic regression

Submitted Feb 26, 2020. Accepted for publication Sep 06, 2020.

doi: 10.21037/atm-20-1960a

View this article at: http://dx.doi.org/10.21037/atm-20-1960a

\section{Introduction}

Hepatic alveolar echinococcosis (HAE) is a potentially fatal disease caused by the larval growth of Echinococcus multilocularis $(1,2)$. HAE, which is also known as "parasitic cancer", frequently manifests as an infiltrating growth similar to that of malignant tumors, leading to the extensive invasion of multiple intrahepatic structures. For HAE, radical hepatectomy accompanied by albendazole therapy is deemed to be the best solution (3). Unfortunately, due to the insidious onset and slow progression of HAE, only a few patients are eligible for conventional radical liver resection, so most patients need to undergo complicated liver resection, even ex vivo liver resection and autotransplantation (ERAT) or liver transplantation (LT) (4-7). The high incidence of postoperative complications after curative liver resection for HAE remains a major issue affecting patient quality of life and prognosis.

Malnutrition is considered to be a preoperative risk factor for adverse postoperative outcomes $(8,9)$. Sarcopenia is also considered a malnutrition syndrome characterized by the progressive loss of skeletal muscle mass and strength and impaired muscular function that is caused by decreased protein synthesis and disturbed energy metabolism (10). Preoperative sarcopenia, muscle steatosis and the accumulation of visceral adipose tissue have been reported to impact surgical complications in some diseases (11-18). Several immunotrophic indices have been considered to be potential predictors of the prognosis of patients after hepatectomy, such as preoperative albumin (ALB), albumin globulin ratio (AGR), red blood cell distribution width (RDW), albumin bilirubin score (ALBI), prognostic nutritional index (PNI), serum creatinine/serum cystatin C (Scre/Scys) ratio (19-26). However, the impacts of the above factors on the postoperative complications of liver resection in HAE patients remain unknown. Furthermore, Pascal et al. found that the existing nutritional screening tools did not show a good prediction ability of the postoperative complications of liver resection in a prospective study (9). Therefore, it is necessary to establish a new nutrition score using other methods to predict complications after liver resection for HAE. This study evaluated the correlation between preoperative skeletal muscle mass and quality, visceral adiposity obtained by radiographic measurements and postoperative complications following liver resection for HAE with data from a large multicenter database. We developed a nomogram to provide clinicians with a quantitative means to assess the individual occurrence of complications after liver resection for HAE and validated the model using other central data to improve clinical decision making. We present the following article in accordance with the TRIPOD reporting checklist (available at http://dx.doi.org/10.21037/atm-20-1960a).

\section{Methods}

\section{Patients}

A retrospective study of HAE patients who underwent curative liver resection between January 2008 and December 2018 was conducted. The diagnosis of HAE was confirmed based on pathological tests and morphological features detected by imaging techniques (27). In addition, we used the clinical data of consecutive patients from 6 hospitals in hydatid-endemic areas who underwent liver resection for HAE in 2019 for external validation. Our selection criteria for patients in this study included the following: (I) pathological diagnosis and imaging findings confirmed HAE; (II) The patient underwent the curative liver resection, which was defined as the complete removal of all macroscopic nodules with a clear margin (R0 resection); and (III) patients were $>18$ years. The exclusion criteria included the following: (I) hepatic cystic echinococcosis (HCE); (II) patients who had a history of malignancy; (III) patients who underwent LT or ERAT; (IV) Patients undergone portal vein embolization two-stage hepatectomy; (V) lack of plain abdominal CT examination within 1 month before the operation, poor clinical data integrity, and (VI) loss to postoperative follow-up within 90 days. The study was conducted in accordance with the Declaration of Helsinki (as revised in 2013). The study was approved by ethics committee of Sichuan University West 


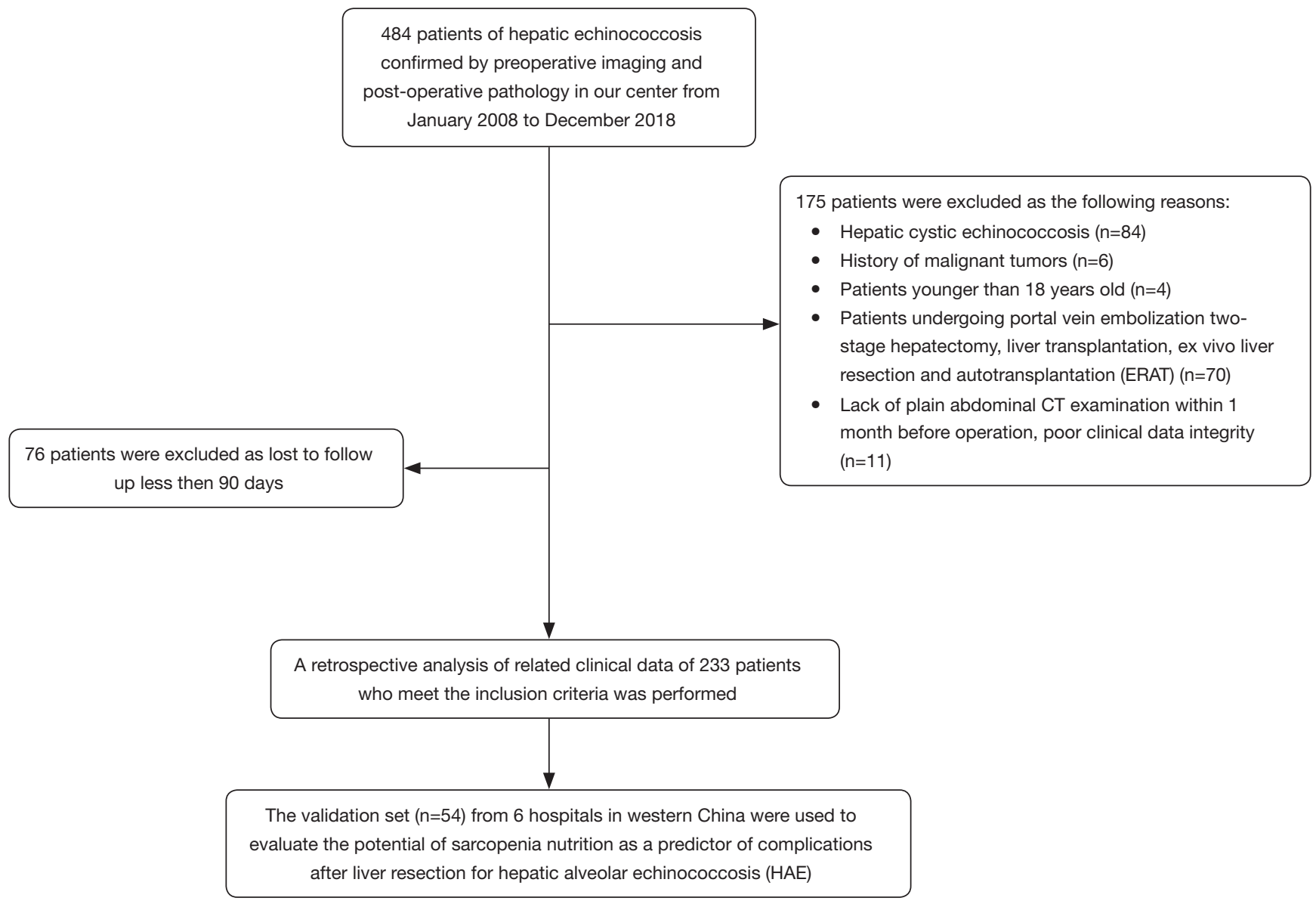

Figure 1 The flowchart of patient selection.

China hospital (2018362) and informed consent was taken from all the patients.

The flowchart of patient selection is shown in Figure 1.

\section{Data collection and image analysis}

The clinicopathological data of patients who underwent curative liver resection for HAE were retrospectively collected from patients' medical records in the hospital and included demographics, preoperative serum biochemistry data, background of liver cirrhosis and characteristics judged by preoperative imaging findings, surgery-related factors, intraoperative blood transfusion (yes or no), the PNM staging system, and postoperative complication outcomes. The liver reserve of all the patients was evaluated using the ALBI classification (ALBI $=\log _{10}$ bilirubin $\times$ $0.66-0.085 \times$ albumin) (21). PNI and AGR were used to assess the nutritional status of the patients. PNI is based on the serum ALB concentration and absolute lymphocyte count, $\mathrm{PNI}=$ serum ALB concentration $(\mathrm{g} / \mathrm{L})+5 \times$ total lymphocyte count $\left(\times 10^{9} / \mathrm{L}\right)(28)$. AGR is defined as the ratio between the serum ALB concentration $(\mathrm{g} / \mathrm{L})$ and serum globulin concentration (g/L) (29). Macroscopic vascular invasion included major hepatic vessel invasion, defined as the invasion of the first-and second-order branches of the portal veins or hepatic arteries, or as the invasion of one or more of the three hepatic veins. According to the cut surface area of the liver after resection and its relationship with the hepatic hilum, the patients were divided into the minor surface area of hepatectomy group and the major surface area of hepatectomy group (30). Post hepatectomy liver failure (PHLF) and bile leakage were defined according to the International Study Group on Liver Surgery (31). The PNM staging system for HAE was developed by the World Health Organization (WHO) (32). Postoperative surgical complications were defined according to the Clavien-Dindo 
classification. Perioperative mortality was defined as patient death within 90 days of surgery (33).

All consecutive plain abdominal computed tomography (CT) images obtained within 1 month before surgery with a 128-row multidetector CT scanner (Somatom Definition Flash; Siemens Medical Systems, Erlangen, Germany) were transferred into our computers. Two doctors (Tao Wang and Xianwei Yang) who had experience in complex image analysis and segmentation techniques performed the parameter measurements and analyzed the cross- sectional non-contrast plain CT images at the level of the third lumbar vertebra using three-dimensional visualization software (Mimics Interactive Medical Image Control System, Version 25.0, Materialize Company, Belgium). We segmented skeletal muscle and adipose tissue and measured the area of skeletal muscle tissue and adipose tissue at the level of the third lumbar vertebra by manually tracing the preoperative plain CT images. Skeletal muscle areas, which included the psoas, erector spinae, quadratus lumborum, transversus abdominis, and external and internal obliques, were identified and quantified using -29 to 150 Hounsfield units (HU) (34). Similarly, adipose tissue areas including subcutaneous adipose tissue and visceral adipose tissue, were quantified using -190 to $-30 \mathrm{HU}$ (34). We used the psoas muscle mass index (PMI), The skeletal muscle mass index (SMI), The visceral to subcutaneous adipose tissue area ratio (VSR), and intramuscular adipose tissue content (IMAC) to reflect the quality and quantity of skeletal muscle, and the degree of visceral fat accumulation. $\mathrm{PMI}=$ cross-sectional areas of left and right psoas muscle $\left(\mathrm{cm}^{2}\right) /$ patient's height ${ }^{2}\left(\mathrm{~m}^{2}\right)$, SMI $=$ cross-sectional areas of skeletal muscle $\left(\mathrm{cm}^{2}\right) /$ patient's height ${ }^{2}\left(\mathrm{~m}^{2}\right)$, and VSR $=$ visceral adipose tissue area $\left(\mathrm{cm}^{2}\right) /$ subcutaneous adipose tissue area $\left(\mathrm{cm}^{2}\right)$. In addition, the quality and myosteatosis of skeletal muscle was examined by IMAC at the L3 level. The CT values of the region of interest (ROIs) of the 4 regions were measured on the subcutaneous fat away from the main blood vessels. The average of these four ROIs was used as the ROI of the subcutaneous fat. Skeletal muscle radiation attenuation (HU) was measured on the multifidus muscles using 2 ROIs. The average of these 2 ROIs was used as the ROI of the multifidus muscles. IMAC $=\mathrm{CT}$ attenuation value of the multifidus muscles (HU)/CT attenuation value of the subcutaneous fat (HU). IMAC indicates a greater amount of adipose tissue within skeletal muscle, and thus a lower quality of skeletal muscle (muscle steatosis). $\mathrm{HU}$ is a measure of the radiation attenuation that can be obtained from a CT scan, as shown in Figure 2.

\section{Statistical analysis}

The Mann-Whitney U test was used to compare continuous variables between the two patient groups. The chi-squared test and two-tailed Fisher's exact test were used for the comparison of categorical variable data between the two groups. Continuous variable data were expressed as medians and ranges, and categorical variable data were expressed as numbers and percentages. The least absolute shrinkage and selection operator (LASSO) logistic regression model was used to build a prognostic classifier for postoperative complications based on sarcopenia in the primary set, which integrated five features out of the seven parameters. Using the coefficients derived from the LASSO logistic regression models, we then constructed a formula to calculate for each patient. We used the receiver operating characteristic (ROC) curve with calculations of the area under the curve (AUC) to determine the optimal cut-off value of the sarcopenia score index. Logistic regression analysis was used to evaluate outcomes based on clinically relevant variables [odds ratio (OR), $95 \%$ confidence interval (CI)]. After univariate analysis, selected variables with $\mathrm{P}$ value $<0.05$ were considered for multivariate regression analysis to investigate the factors related to postoperative complications. In the multivariate regression model, the $\mathrm{P}$ value was set at 0.05 . A nomogram incorporating sarcopenia score index and risk factors assessed based on multivariable logistic regression was constructed for predicting postoperative complications. The predictive accuracy of the models was measured using the C-index, which quantifies the level of agreement between the predicted probabilities and the actual probabilities of having the event of interest, and the bootstrap estimate of slope shrinkage (35). In addition, we used clinical data from 6 hospitals in hydatidendemic areas for external validation. During the external validation, the total points of each patient in the validation set were calculated according to the established nomogram, and the C-index and calibration curve were derived based on the regression analysis. Decision curve analysis (DCA) was performed to determine the clinical application value of the nomogram models by calculating the net benefits at each risk threshold probability. All analyses were conducted using SPSS statistical software version 24.0 (IBM Corporation, Armonk, NY, USA), GraphPad Prism 8 and R version 3.61(http://www.r-project.org/). 

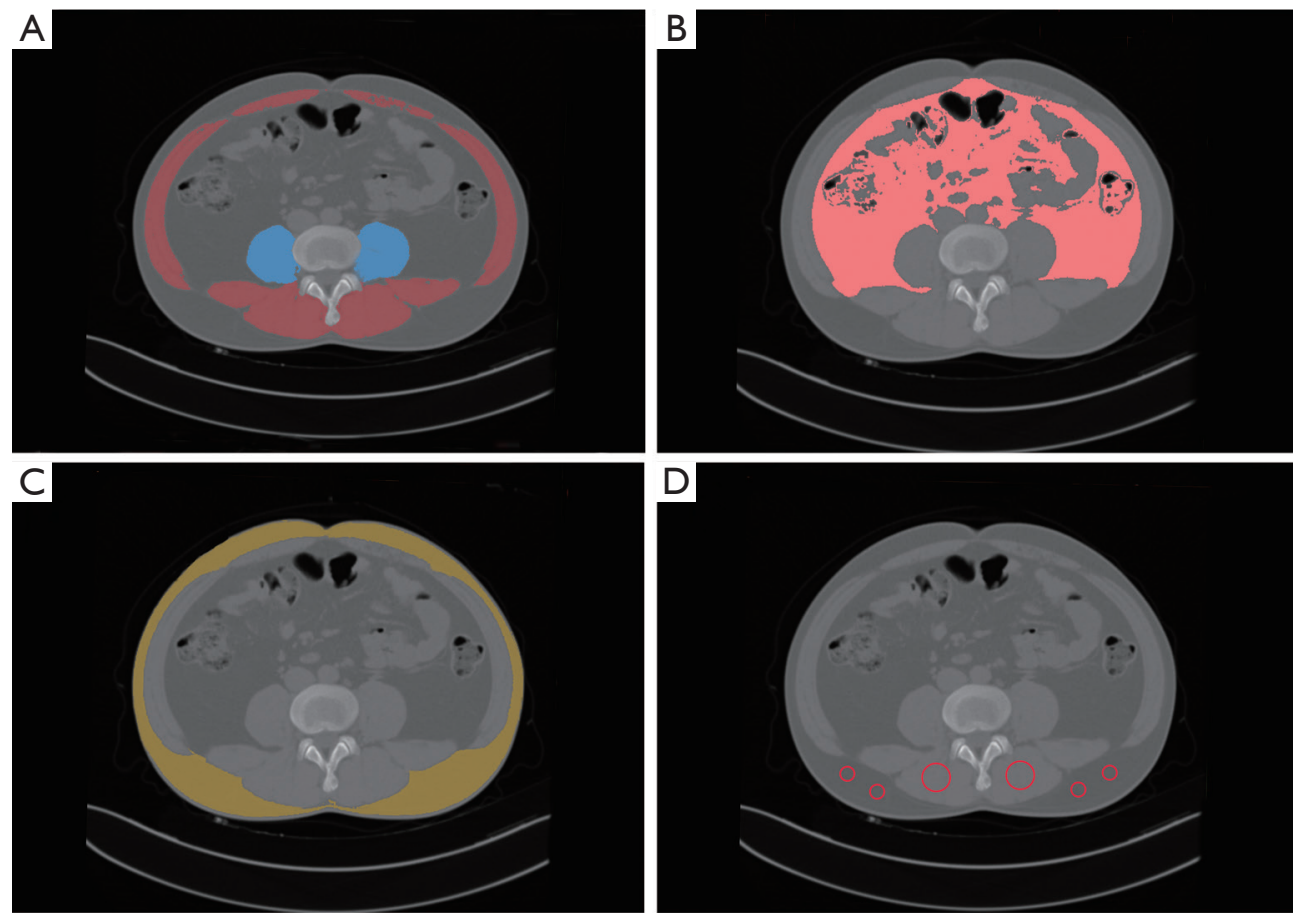

Figure 2 Cross-sectional computed tomography (CT) images at the level of the third lumbar vertebra (L3). (A) Skeletal muscle areas are quantified using a CT attenuation value of -29 to 150 Hounsfield units (HU). (B) Visceral adipose tissue areas were quantified using attenuation values of -150 to $-50 \mathrm{HU}$. (C) Subcutaneous adipose tissue areas were quantified using attenuation values of -190 to $-30 \mathrm{HU}$.

(D) The quality and myosteatosis of skeletal muscle were examined by intramuscular adipose tissue content (IMAC) at the L3 level. The

CT values of subfascial muscular tissue in the multifidus muscle (2 circles) and subcutaneous fat ( 4 small circles) were examined to calculate IMAC.

\section{Results}

\section{Patient characteristics}

In the primary set, a total of 233 patients, including 124 male and 109 female patients, with an age range of 18 to 69 years, confirmed by pathology who underwent curative liver resection were eligible for this study. The validation set included 54 patients from 6 hospitals in hydatid-endemic areas. The clinicopathologic characteristics of the patients in the primary and validation sets are listed in Table 1 . In the primary set, a total of 82 patients $(82 / 233,35.2 \%)$ experienced postoperative complications during the study period with a major postoperative complication rate (ClavienDindo $\geq 3$ ) of $18.02 \%$ (42/233) (33). The postoperative complications after liver resection included wound infection $(n=14)$, bile leakage $(n=19)$, intra-abdominal abscess $(n=11)$, hydrothorax $(\mathrm{n}=16)$, gastroparesis $(\mathrm{n}=11)$, intra-abdominal hemorrhage $(n=2)$, ascites $(n=17)$, respiratory infection $(n=20)$ and acute liver or renal failure $(\mathrm{n}=2)$.

\section{Prognostic nomogram for predicting complications after liver resection for $H A E$}

Using the coefficients derived from the LASSO logistic regression models based on the primary set, we then constructed a formula to calculate for each patient. The LASSO coefficient profiles of the selected blood features are shown in Figure 3. The sarcopenia score is based on their personalized levels of the five features, where sarcopenia score $=2.091+1.109 \times \mathrm{VSR}+0.797 \times \mathrm{IMAC}$ $-0.016 \times$ Age $-0.374 \times$ PMI $-0.019 \times$ SMI. Using ROC curves, we classified patients into a sarcopenia type A and sarcopenia type B group with a nutritional score of -1.166 as the cut-off value. In the primary set, 63 patients $(63 / 233$, $27 \%)$ were classified into the sarcopenia type A group. The remaining 170 patients (73\%) were classified into the sarcopenia type B group. No significant differences in the baseline characteristics were observed between the groups (all $\mathrm{P}>0.05)$, except for the sex variable (19/44 vs.105/65, $\mathrm{P}<0.001)$, hemoglobin $(\mathrm{HGB})$ variable $(130$ vs. $137.5 \mathrm{~g} / \mathrm{L}$, 
Table 1 Perioperative data

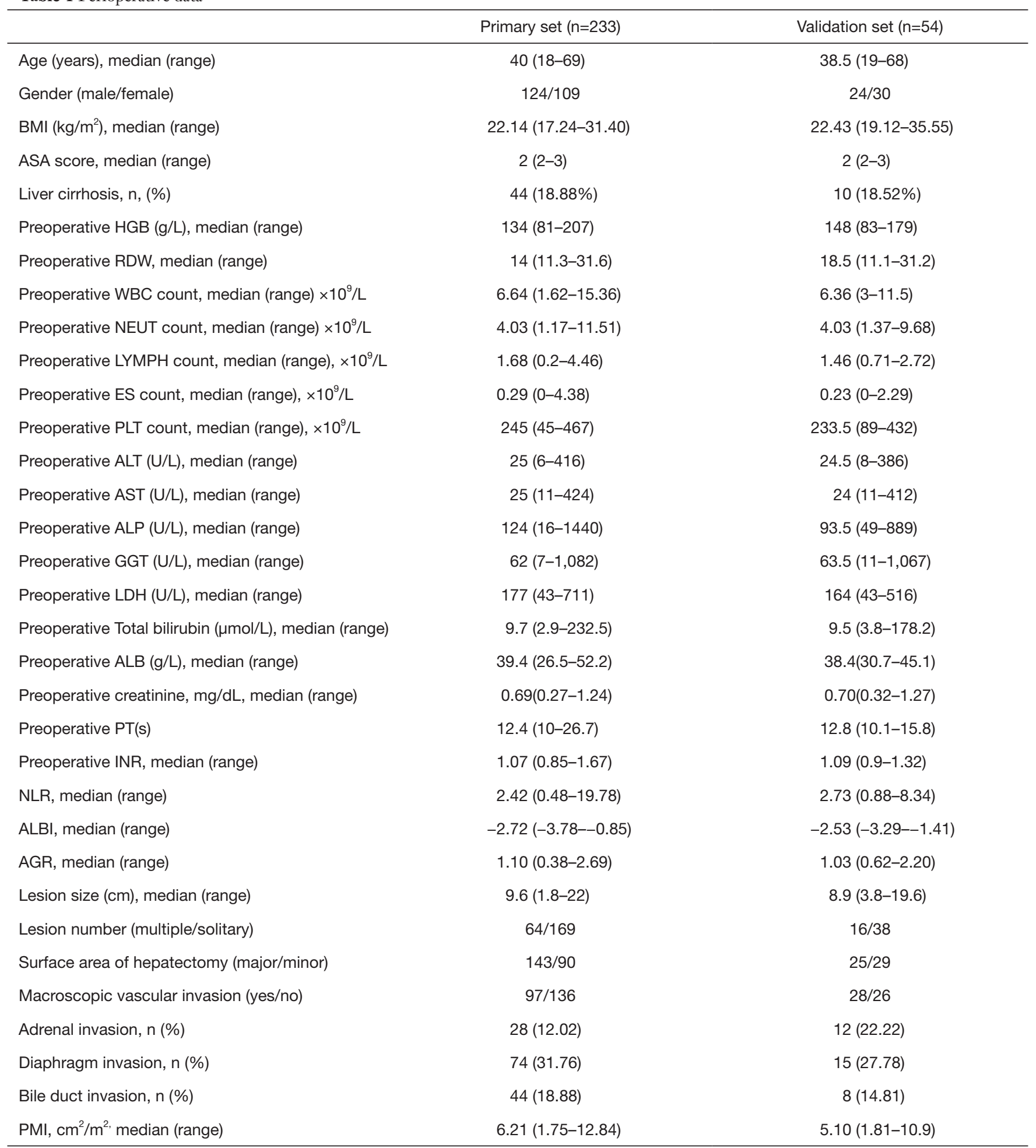

Table 1 (continued) 
Table 1 (continued)

\begin{tabular}{lcc}
\hline & Primary set $(\mathrm{n}=233)$ & Validation set $(\mathrm{n}=54)$ \\
\hline $\mathrm{SMI}, \mathrm{cm}^{2} / \mathrm{m}^{2}$, median (range) & $42.76(23.88-67.16)$ & $42.14(25.09-67.52)$ \\
VSR, $\mathrm{cm}^{2} / \mathrm{cm}^{2}$, median (range) & $0.35(0.05-2.90)$ & $0.28(0.07-2.34)$ \\
IMAC, median (range) & $-0.54(-1.89--0.06)$ & $-0.62(-1.18--0.23)$ \\
Surgical approach (LLR/OLR) & $11 / 222$ & $1 / 53$ \\
Transfusion (yes/no) & $54 / 179$ & $15 / 39$ \\
PNM stage (early stage/late stage) & $154 / 79$ & $34 / 20$ \\
Hospital stay (days) & $12(6-66)$ & $23.5(14-45)$ \\
Postoperative complication (Clavien-Dindo $\geq 3)$ & $42(18.02 \%)$ & $13(24.07 \%)$ \\
Postoperative 90-day mortality & $4(1.72 \%)$ & $1(1.85 \%)$ \\
\hline
\end{tabular}

BMI, body mass index; ASA, American Society of Anesthesiology; HGB, hemoglobin; RDW, Red blood cell distribution width; WBC, white blood cell; NEUT, neutrophil; LYMPH, lymphocyte: MONO, Monocyte; ES, eosinophil; PLT, platelets; ALT, alanine aminotransferase; AST, aspartate transaminase; ALP, alkaline phosphatase; GGT, $\gamma$-glutamyl transferase; LDH, lactate dehydrogenase; PT, Prothrombin time; INR, international normalized ratio; NLR, neutrophil-to-lymphocyte; ALBI grade, albumin-bilirubin grade; AGR, albumin/globulin ratio; LLR, laparoscopic liver resection; OLR, open liver resection; PMI, psoas muscle mass index; SMI, Skeletal muscle mass index; VSR, visceral to subcutaneous adipose tissue area radio; IMAC, intramuscular adipose tissue content.
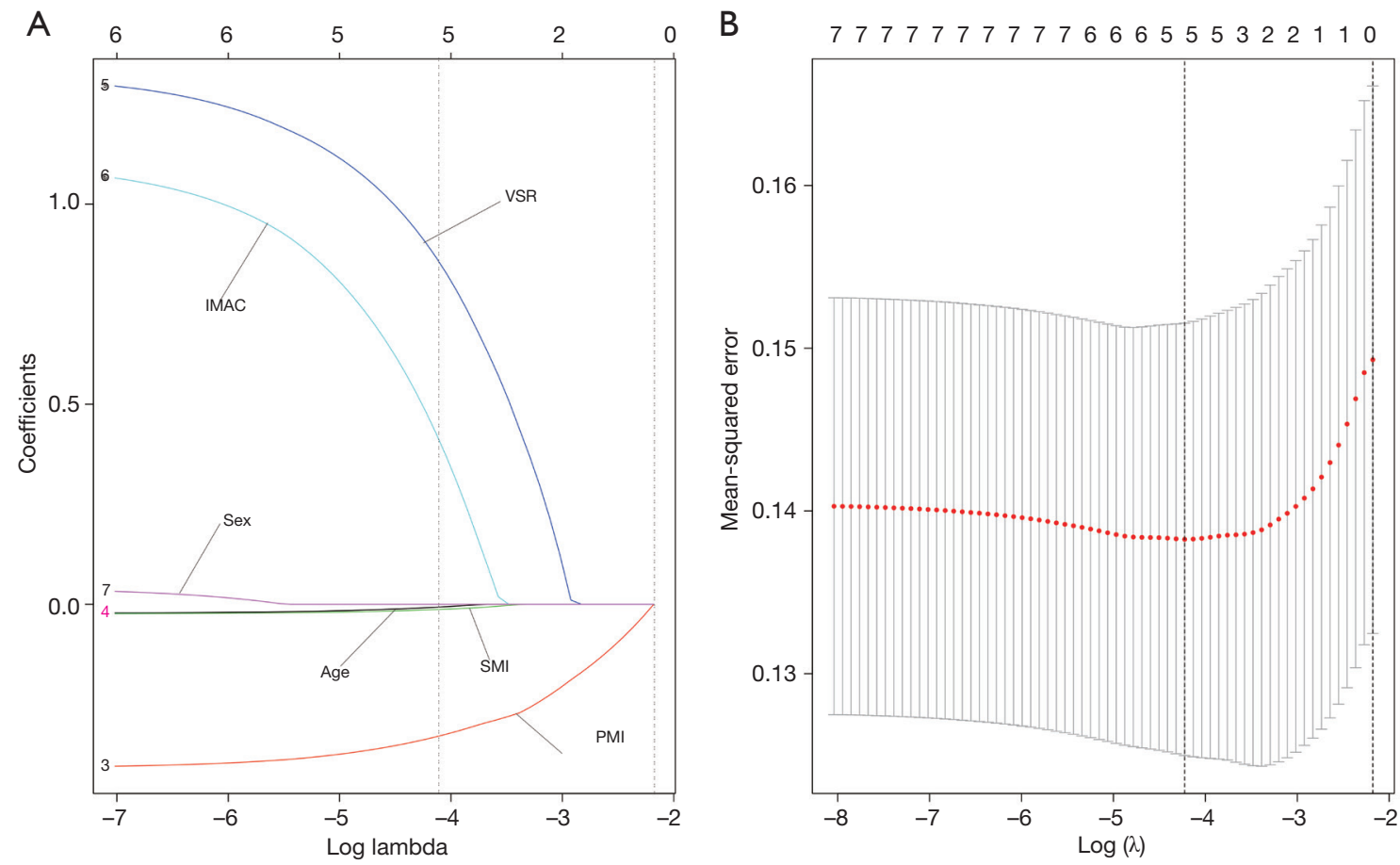

Figure 3 (A) The least absolute shrinkage and selection operator (LASSO) coefficient profiles of the five selected sarcopenia features. A dashed vertical line is drawn at the value $(\log \gamma=-4.3)$ chosen by 10 -fold cross-validation. (B) Partial likelihood deviance for the LASSO coefficient profiles. A light dashed vertical line stands for the minimum partial likelihood deviance. A dashed vertical line stands for the partial likelihood deviance at the value $(\log \gamma=-4.3)$. 
$\mathrm{P}=0.006)$, white blood cell $(\mathrm{WBC})$ count variable $\left(6.07 \times 10^{9}\right.$ vs. $\left.6.745 \times 10^{9} / \mathrm{L}, \mathrm{P}=0.017\right)$, total bilirubin variable (7.8 vs. $10.15 \mu \mathrm{mol} / \mathrm{L}, \mathrm{P}=0.036$ ), $\mathrm{PMI}$ variable (4.18 vs. $\left.7.04 \mathrm{~cm}^{2} / \mathrm{m}^{2}, \mathrm{P}<0.001\right)$, SMI variable (37.02 vs. 45.01 $\left.\mathrm{cm}^{2} / \mathrm{m}^{2}, \mathrm{P}<0.001\right)$, VSR variable $\left(0.40\right.$ vs. $0.32 \mathrm{~cm}^{2} / \mathrm{cm}^{2}$, $\mathrm{P}=0.026)$, IMAC variable $(-0.51$ vs. $-0.56, \mathrm{P}<0.001)$ and hospital stay ( $14 v s .11 .5$ days, $\mathrm{P}=0.001)$. In addition, patients with sarcopenia type A had a significantly different 90 day mortality rate $(4.76 \%$ vs. $0.59 \% ; \mathrm{P}=0.029)$, as listed in Table 2. According to univariable logistic regression analysis, sex, preoperative eosinophils (ES), preoperative alkaline phosphatase (ALP), preoperative $\gamma$-glutamyl transferase (GGT), preoperative lactate dehydrogenase (LDH), ALBI, maximum lesion size, blood transfusion (yes vs. no), macroscopic vascular invasion (yes vs. no), adrenal invasion (yes $v s$. no), surface area of hepatectomy (major $v s$. minor) increased the odds of major postoperative major complications. These variables were included in a multivariable logistic model, and the multivariate analyses revealed that preoperative GGT $\geq 63.5 \mathrm{U} / \mathrm{L}$ (OR, 2.527, 95\% CI, 1.013-6.301, $\mathrm{P}=0.047$ ), the major surface area of hepatectomy (OR, 3.210, 95\% CI, 1.064-9.687, $\mathrm{P}=0.038$ ) were identified as independent risk factors for postoperative complications (Table 3, Figure 4). Based on the results of the multivariate logistic analysis and LASSO logistic analysis, a nomogram integrating all significant independent factors was constructed to predict postoperative complications for HAE patients after liver resection (Figure $5 A$ ). The model had a good prediction capability with a C-index of 0.84 (95\% CI, 0.72-0.96). In the validation set, the C-index of the nomogram for predicting postoperative complications was 0.92 (95\% CI, 0.85 to 0.99 ), The calibration plot for the probability of postoperative complications showed an optimal agreement between the nomogram prediction and the actual observation in the primary and validation sets (Figure 5B, 5C). The decision curves of the nomograms for predicting postoperative complications are presented in the primary set and validation set are shown in Figure 6. In addition, in this research, we also analyzed the correlation between sarcopenia score and some indicators related to inflammation and nutritional metabolism. We found that the sarcopenia score was correlated with WBC count ( $\mathrm{r}$ $=-0.206, \mathrm{P}=0.020)$, RDW ( $\mathrm{r}=0.151, \mathrm{P}=0.021)$, ALB level $(\mathrm{r}$ $=-0.240, \mathrm{P}<0.001), \mathrm{Scr} / \mathrm{Scys}(\mathrm{r}=-0.259, \mathrm{P}=0.002)$ and $\mathrm{PNI}$ score $(\mathrm{r}=-0.152, \mathrm{P}=0.020$; Figure $\mathrm{S} 1)$.

\section{Discussion}

Although HAE is regarded as a benign disease, due to the long-term growth of HAE lesions in the body and the trait of invasion along blood vessels and biliary structures, the lesions of HAE that exhibits tumor-like characteristics are typically large, multiple, or infringing on adjacent structures, and the first and second hepatic portal veins are involved at the first diagnosis $(2,32)$. Surgical curative liver resection still remains the main treatment for endstage HAE $(24,36)$. While advances in surgical technique and perioperative management for HAE have dramatically decreased postoperative mortality and improved the clinical cure rate. However, due to the complexity of liver resection for HAE, the incidence of postoperative complications is still high $(30,36)$. Severe postoperative complications would prolong the length of hospital stay, increase hospital costs, increase the probability of readmission and even worsen the long-term prognosis $(24,37)$. Therefore, it is necessary to identify the risk factors for increased postoperative complications after liver resection for HAE.

Recent studies have shown that sarcopenia and visceral adiposity, as a more and more closely watched predictor, play important roles in nutritional assessment, which has been proven to be related to postoperative complications in various diseases including gastric cancer, hepatocellular carcinoma (HCC), pancreatic cancer, and endometrial cancer (38-42). However, the impacts of preoperative sarcopenia, muscle steatosis, and visceral adiposity on short-term outcomes after liver resection for HAE remain unclear. This retrospective study was the first comprehensive study to show that sarcopenia and visceral adiposity were significant predictors of postoperative major complications following liver resection for HAE. Distinct from other studies that focused on only the effects of sarcopenia or visceral fat on complications after liver resection, we utilized the LASSO logistic regression model $(\log \gamma=-4.3)$ to select the most useful prognostic parameters for sarcopenia score(combining with sarcopenia and visceral adiposity), and then established a classifier based on multimuscle nutrition features for predicting postoperative complications in the primary and validation sets. After adjustment for confounding factors, our sarcopenia classifier was an independent prognostic factor for postoperative complications with good predictive accuracy in the primary and validation set. Notably, our sarcopenia classifier which 


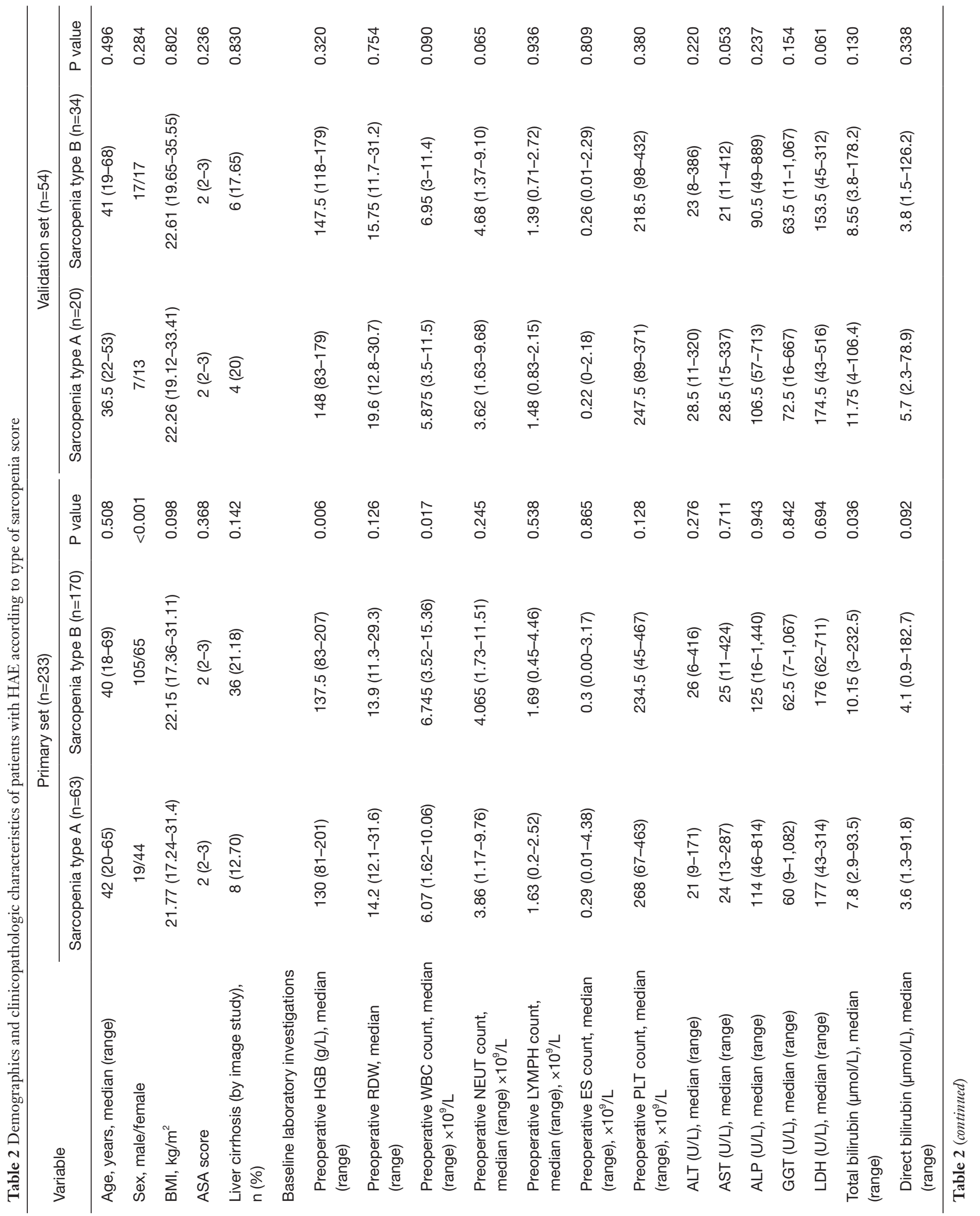




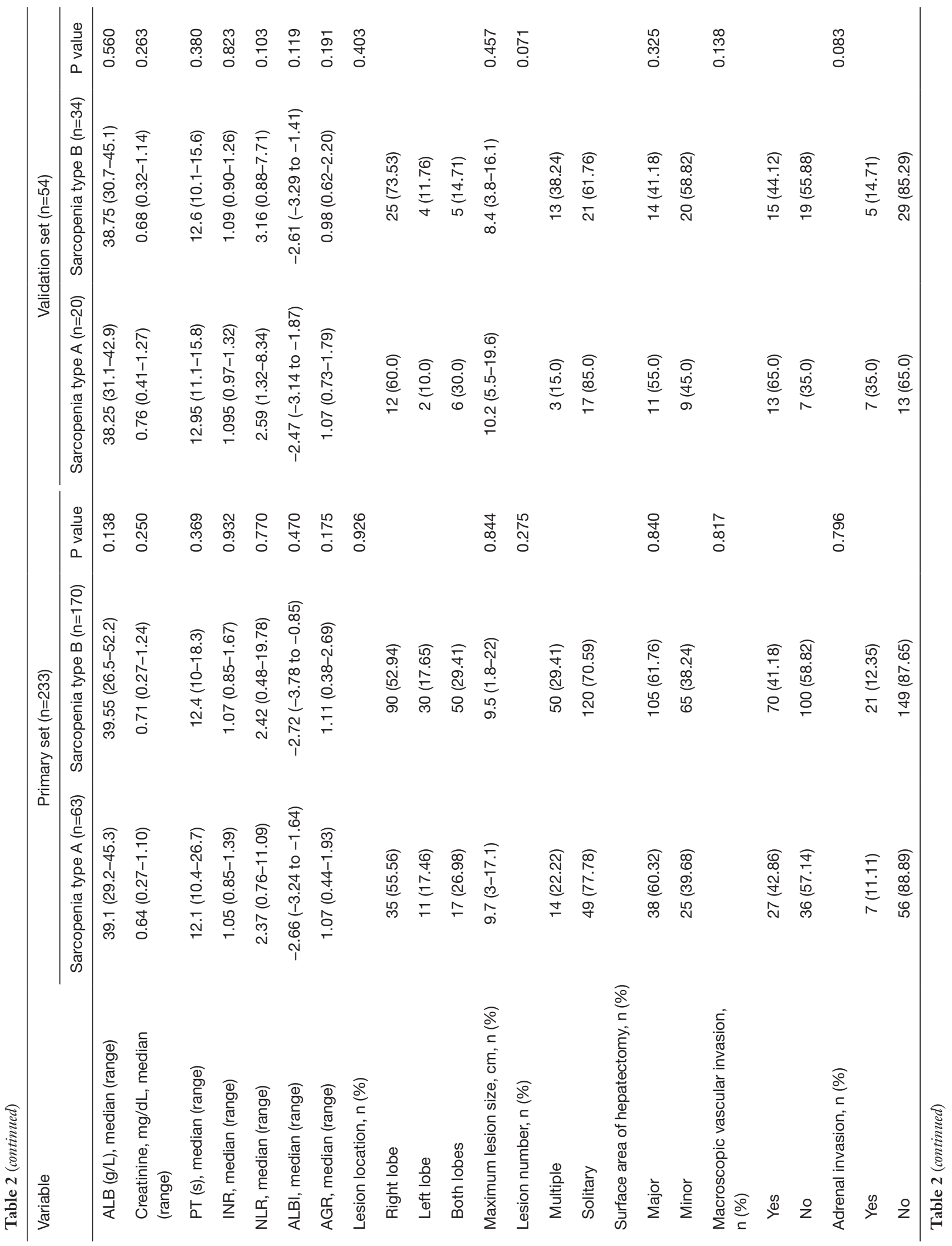




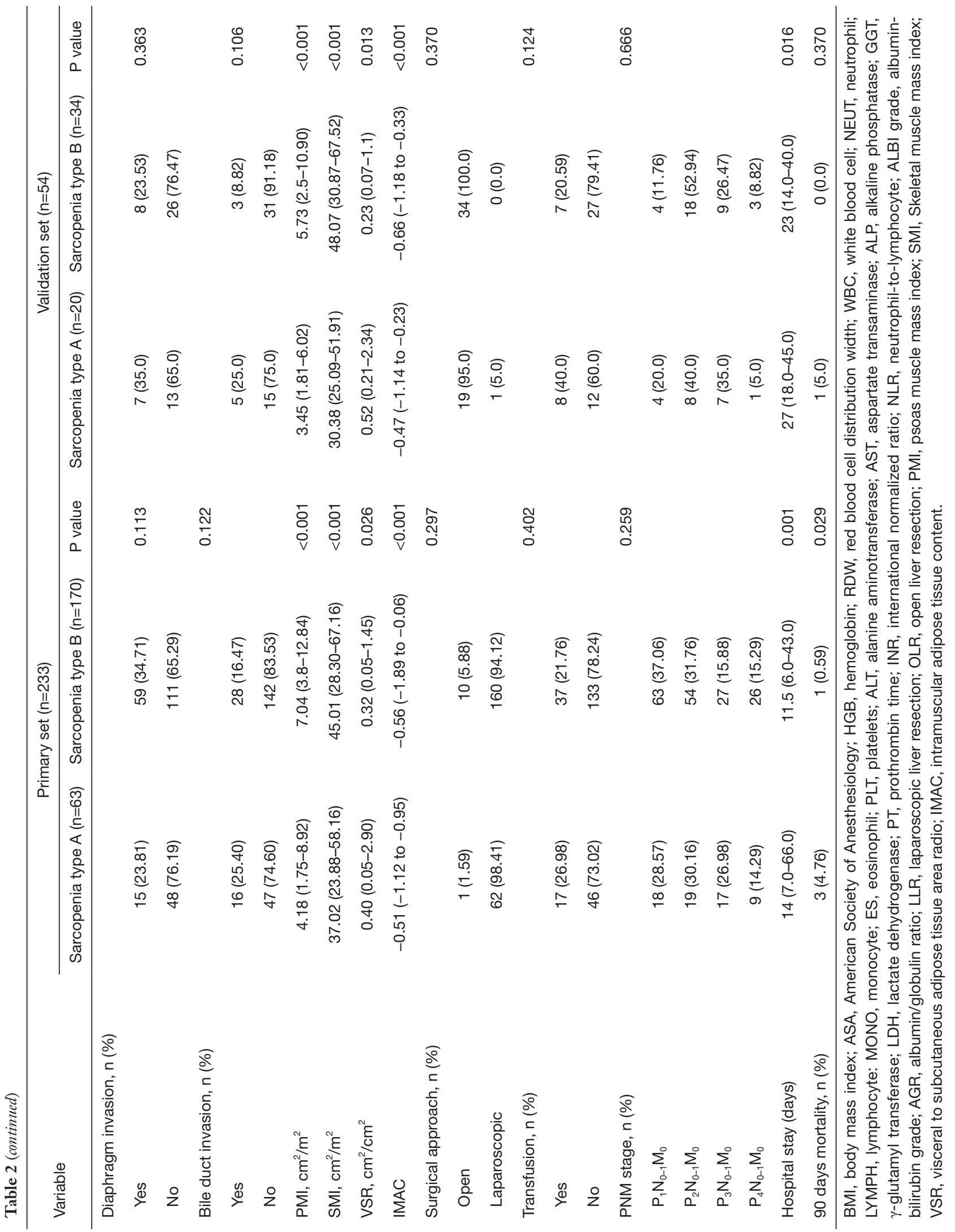


Table 3 Logistic regression models of variables associated major postoperative complications

\begin{tabular}{|c|c|c|c|c|c|c|}
\hline Variable & \multicolumn{3}{|c|}{ Univariate regression model } & \multicolumn{3}{|c|}{ Multivariate regression model } \\
\hline Age ( $\geq 40$ vs. $<40$ years) & 0.777 & $0.398-1.516$ & 0.459 & 0.858 & $0.405-1.819$ & 0.690 \\
\hline Sex (female vs. male) & 2.114 & $1.066-4.196$ & 0.032 & 2.041 & $0.937-4.445$ & 0.073 \\
\hline Liver cirrhosis (yes vs. no) & 1.443 & $0.648-3.215$ & 0.370 & & & \\
\hline Preoperative ALP ( $\geq 164.5$ vs. $<164.5$ ) & 2.720 & $1.376-5.378$ & 0.004 & 1.085 & $0.442-2.663$ & 0.859 \\
\hline Preoperative GGT ( $\geq 63.5$ vs. <63.5) & 3.253 & $1.571-6.737$ & 0.001 & 2.527 & $1.013-6.301$ & 0.047 \\
\hline Preoperative LDH ( $\geq 186.5$ vs. $<186.5$ ) & 2.646 & $1.339-5.228$ & 0.005 & 1.782 & $0.801-3.966$ & 0.157 \\
\hline Preoperative ALB ( $\leq 30$ vs. $>30$ g/dL) & 1.905 & $0.568-6.396$ & 0.297 & & & \\
\hline $\mathrm{PNI}(\leq 47$ vs. $>47)$ & 1.680 & $0.858-3.290$ & 0.131 & & & \\
\hline Scre/Scys ( $\geq 1.27$ vs. $<1.27)$ & 2.372 & $0.569-9.894$ & 0.236 & & & \\
\hline Maximum lesion size (>10 vs. $\leq 10 \mathrm{~cm}$ ) & 2.393 & $1.196-4.785$ & 0.014 & 0.638 & $0.248-1.640$ & 0.351 \\
\hline Lesion number (multiple vs. single) & 0.567 & $0.247-1.302$ & 0.181 & & & \\
\hline Blood loss $>500 \mathrm{~mL}$ & 0.993 & $0.951-1.036$ & 0.736 & & & \\
\hline Blood transfusion (yes vs. no) & 2.165 & $1.051-4.460$ & 0.036 & 1.351 & $0.582-3.135$ & 0.484 \\
\hline Macroscopic vascular invasion (yes vs. no) & 3.457 & $1.735-6.891$ & $<0.001$ & 1.802 & $0.788-4.121$ & 0.163 \\
\hline Bile duct invasion (yes vs. no) & 1.987 & $0.921-4.291$ & 0.080 & & & \\
\hline
\end{tabular}

ES, eosinophil; ALP, alkaline phosphatase; GGT, $\gamma$-glutamyl transferase; LDH, lactate dehydrogenase; ALB, albumin; NLR, neutrophil-tolymphocyte ratio; ALBI grade, albumin-bilirubin grade; PNI, prognostic nutrition index; Scre/Scys, serum creatinine/serum cystatin C.

was established from preoperative plain CT scans, was an objective clinical quantification of a patient's nutritional status, fitness level, and muscle frailty by reflecting the degenerative loss of skeletal muscle mass and increase in visceral fat. This classifier can be used in clinical practice and can potentially guide clinical decisions and informed consent prior to surgical liver resection based on the parameters.

Studies have reported that sarcopenia might be related to the activation of the ubiquitin proteasome pathway and caspases under transcriptional control of the transcription factors forkhead box $\mathrm{O}$ (Fox-O) and nuclear factor (NF)$\kappa \mathrm{B}$, Moreover, myostatin, which is a member of the transforming growth factor $\beta$ (TGF $\beta$ ) family, can also cause myopenia by down regulating the mammalian target of rapamycin (mTOR) pathway (43-45). However, the precise mechanism of how low skeletal muscle mass with increasing visceral adipose tissue affects postoperative complications is unclear. One possible mechanism is that sarcopenia may reduce muscle strength, leading to poor physical function and thereby reducing tolerance to surgical liver resection treatment (46). In addition, due to the decrease in the amount of protein stored in the body due to sarcopenia, metabolism and immunity are also reduced, and cause an imbalance between adipokines and myokines, which can easily lead to an immune deficiency state for adding surgical 

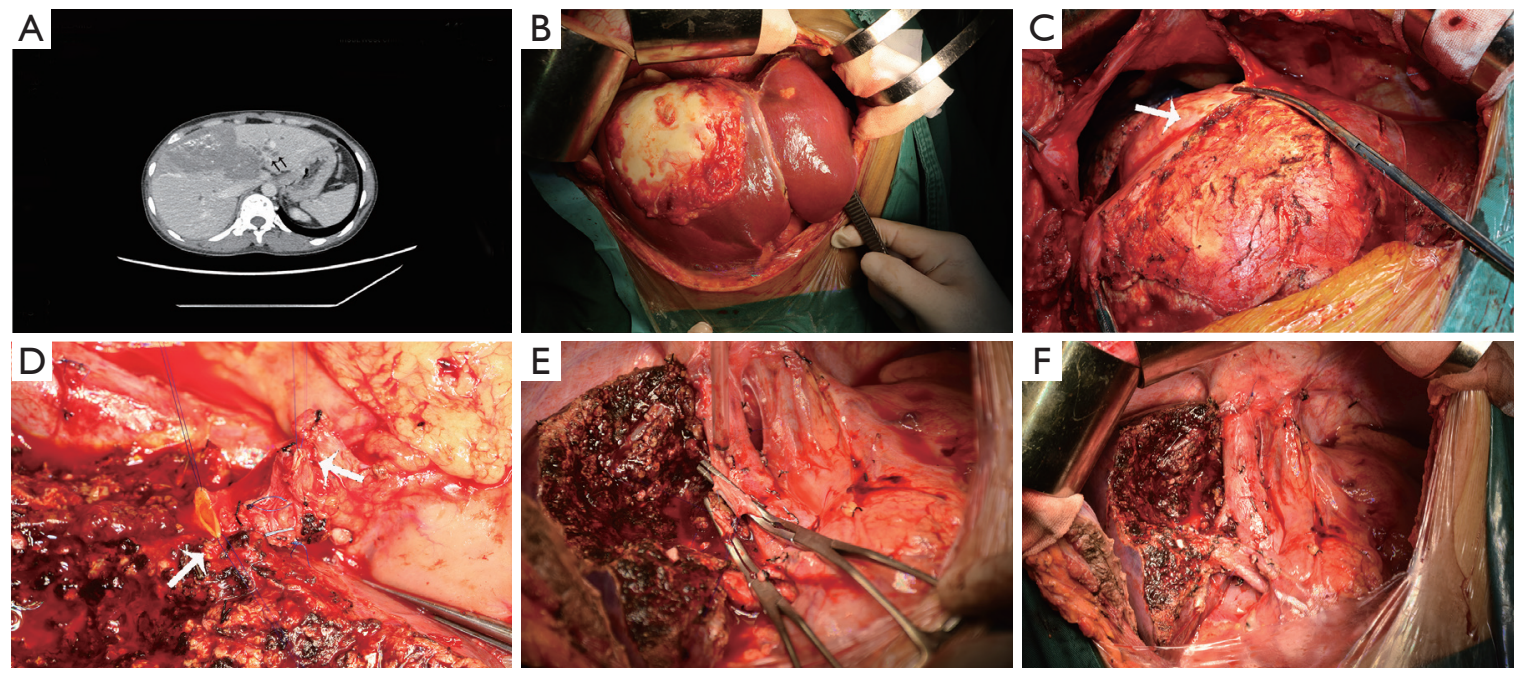

Figure 4 Preoperative assessment and surgical techniques. (A, B) A preoperative computed tomography (CT) scan showed a massive lesion of hepatic alveolar echinococcosis (HAE) occupying the left medial lobe and right anterior lobe, resulting in left hepatic bile duct dilation (black arrow). (C) A HAE lesion in the right liver involving the diaphragm (white arrow). (D) A HAE lesion invading liver parenchyma and biliary tract. After complete removal of the HAE lesion, then anastomosis of the biliary tract was performed (white arrow). (E) A HAE lesion invading the right posterior inferior vein of the liver. After complete removal of the HAE lesion, the right posterior inferior vein was reconstructed. (F) A larger surface area of liver resection after the HAE lesion was removed.

intervention and the dysfuction of postoperative liver cells $(40,47)$, which in turn leads to an increase in the incidence of postoperative complications and postoperative mortality.

Similarly, adipose tissue plays an important role in the prognosis after liver resection. Adipose tissue not only affects the endocrine system but is also a key part of the immune system. Some research has proven that excess visceral fat has more metabolic activity than subcutaneous fat and that the accumulation of visceral adipose tissue can lead to the secretion of various kinds of proinflammatory adipokines including leptin, tumor necrosis factor- $\alpha$ (TNF- $\alpha$ ), interleukin (IL)-1, IL-6, monocyte chemoattractant protein (MCP)-1, and to a decrease in the amount of anti-inflammatory adipokines, such as adiponectin, which lead to a chronic proinflammatory state and even contribute to insulin resistance (48-50). Although the inflammatory response provides a protective effect for the body against trauma or infection, chronic inflammation can be maladaptive and may potentiate the proinflammatory response to the trauma induced by surgery (51). In our research, we find found that the sarcopenia score was correlated with WBC, RDW, ALB, PNI, and Scr/Scys, which are important indicators related to inflammation and nutritional metabolism. Poor nutrition associated with the inflammatory response and low nutritional immunosuppressive status. Preoperative intervention to improve body composition such as adequate protein (branched-chain amino acids) or aerobic exercise might lead to improved prognosis after liver resection $(45,52)$. More prospective studies are still needed to further explore the relationship between cytokines and visceral adipose or sarcopenia.

In our research, preoperative GGT levels were found to be independent risk factors for major postoperative complications, which was consistent with previous research findings $(53,54)$. Serum GGT is related to the accumulation of bile in capillaries caused by chronic biliary infection and immune factors. Serum GGT has been proposed as a sensitive landmark of oxidative stress, which is related to the production of reactive oxygen species (ROS) (55). High serum GGT levels might reflect the severity of the damage that HAE causes to the liver in an inflammatory response. In addition, the larger the area of hepatectomy is, the more complicated the hepatectomy becomes, the longer the operation time is, and the greater the damage to the body will be. Individualized surgical design was needed to perform according to the size and location of the lesion to avoid bleeding and unnecessary cutting of the liver surface area to ensure the safety of surgery and reduce postoperative complications. 
Page 14 of 18

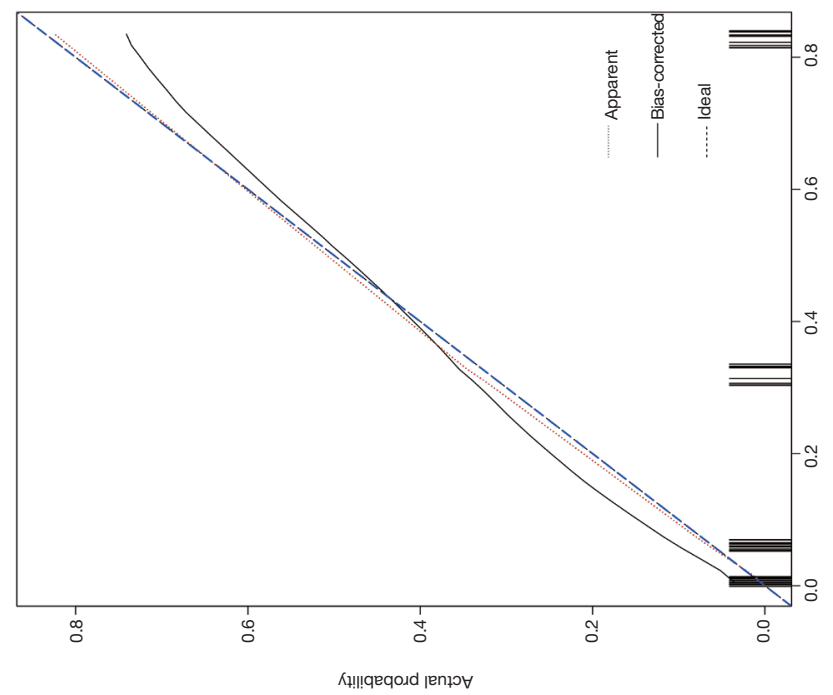

$\cup$

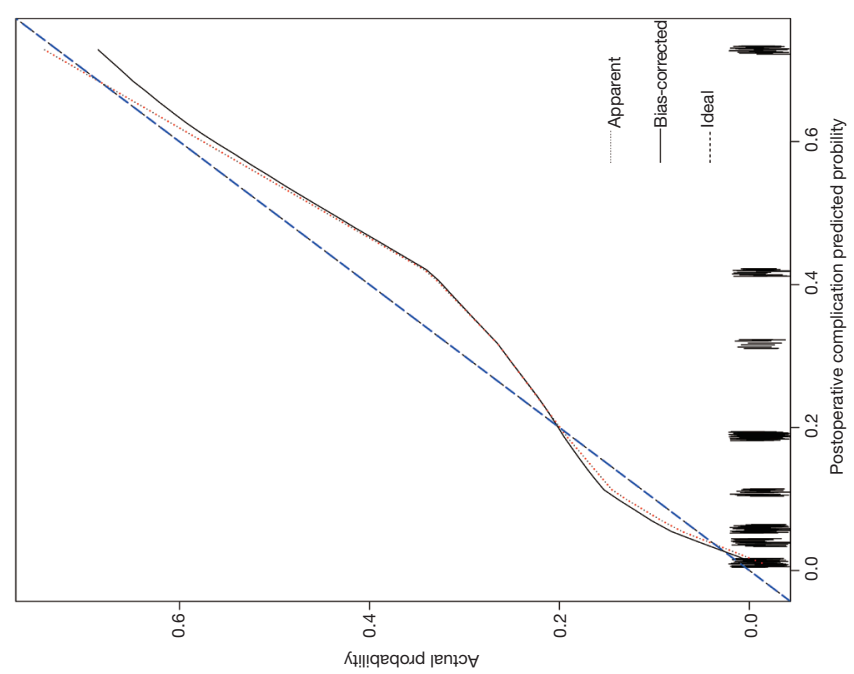

$\infty$

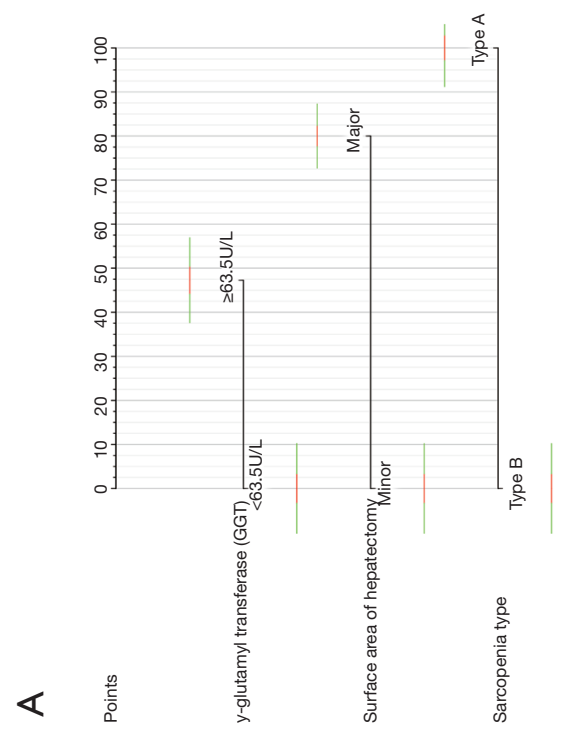

Wang et al. A new sarcopenia score prognostic for PCs for HAE

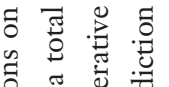

$\pi$ 范

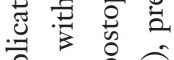

象

ए

焉

㻤

要过

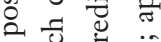

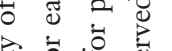

氜茧客

ลे

ํㅠㄹ च

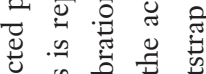

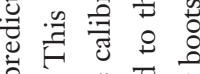

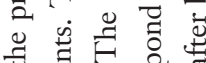

क ¿ं की

药

콘

क $=\overline{0}$

ป ป ฐ

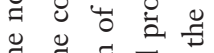

Е ฐ छ चु

全言道总

ப.

䒕

. ฮี

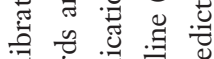

䒕 䒕

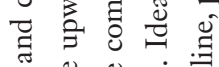

苛.

.

氙.

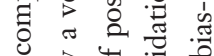

웡

氙要要

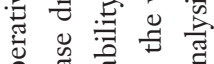

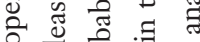

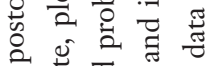

.

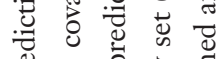

苟

ธั

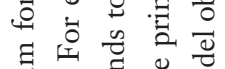

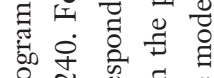

‡

乙。

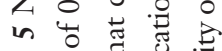

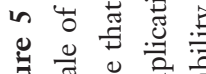

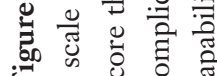


A

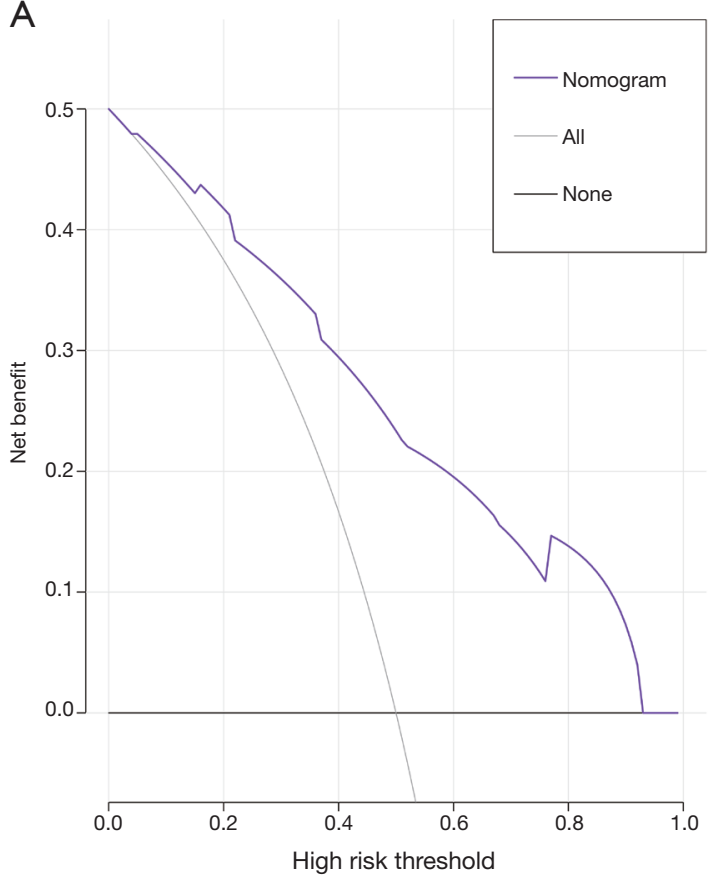

B

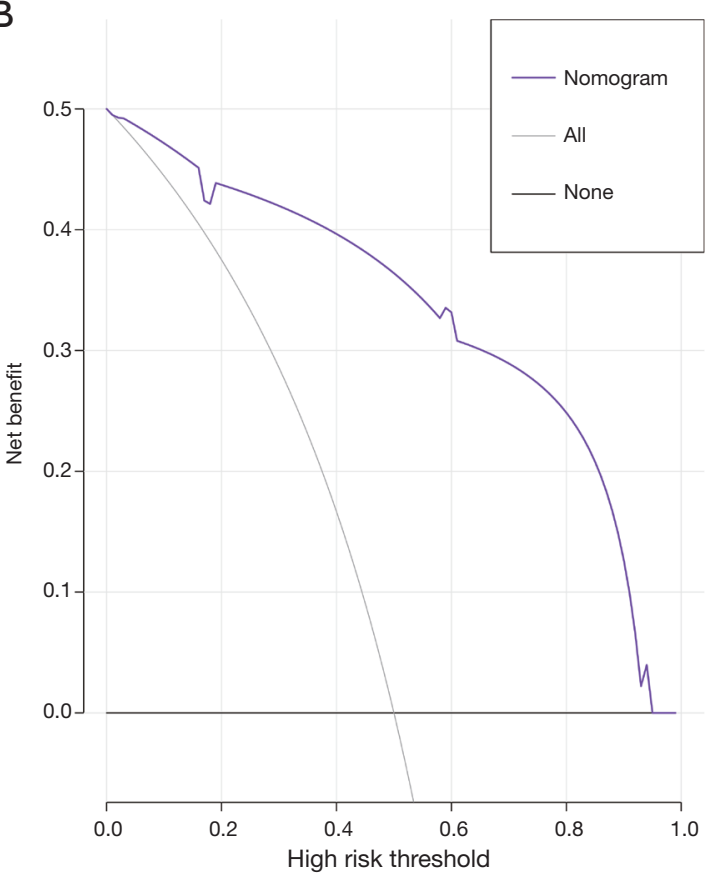

Figure 6 The decision curves of the nomograms for predicting postoperative complications in the primary set and validation set are presented.

Through the nomogram we established, we could offer clinicians a simple and quantitative means to assess the individual probability of postoperative complications of each HAE patient before surgery. For the high-risk population, we can choose a combination of nutritional support treatment and resistance training first. After improving the nutritional status and physical fitness of patients, we can choose surgical treatment to improve the short-term prognosis of patients; However, we still need prospective studies or randomized controlled studies to confirm the prognostic benefits of nutritional or physical fitness improvement on patients with HAE.

As a retrospective study, our study had several inherent limitations. First, the nomogram we established for predicting for postoperative complications was based on data obtained from a single HAE center in China. Although we used other data from multiple hospitals in western China for verification, a pooled analysis with a database from different centers in different districts certainly introduces some selection bias. Second, the mechanisms behind the associations of sarcopenia and postoperative major complications were not clearly elucidated in our study, and further investigations may provide more information for a better understanding of the roles of nutrition (including sarcopenia and visceral adiposity) on postoperative complications for HAE.

In conclusion, our study showed that sarcopenia score evaluated by PMI, SMI, IMAC and VSR was significantly correlated with postoperative major complications in patients undergoing curative liver resection for HAE. In addition, the preoperative nutritional intervention and rehabilitation could be important for good outcomes after liver resection.

\section{Acknowledgments}

Funding: This research was founded by the National Natural Science Foundation of China (No. 81770566), the Department of Science and Technology of Sichuan Province (No. 19ZDYF1682), State Key Laboratory of Pathogenesis, Prevention and Treatment of High Incidence Disease in Central Asia (SKL-HIDCA-2017-14), Inner Mongolia Autonomous Region Applied Technology Research and Development Funding Plan (No. Kjt15sf05).

\section{Footnote}

Reporting Checklist: The authors have completed the 


\section{Page 16 of 18}

TRIPOD reporting checklist. Available at http://dx.doi. org/10.21037/atm-20-1960a

Data Sharing Statement: Available at http://dx.doi. org/10.21037/atm-20-1960a

Conflicts of Interest: All authors have completed the ICMJE uniform disclosure form (available at http://dx.doi. org/10.21037/atm-20-1960a). The authors have no conflicts of interest to declare.

Ethical Statement: The authors are accountable for all aspects of the work in ensuring that questions related to the accuracy or integrity of any part of the work are appropriately investigated and resolved. The author confirmed that the figure(s) and table(s) appearing in this manuscript are originality. The study was conducted in accordance with the Declaration of Helsinki (as revised in 2013). The study was approved by ethics committee of Sichuan University West China hospital (2018362) and informed consent was taken from all the patients.

Open Access Statement: This is an Open Access article distributed in accordance with the Creative Commons Attribution-NonCommercial-NoDerivs 4.0 International License (CC BY-NC-ND 4.0), which permits the noncommercial replication and distribution of the article with the strict proviso that no changes or edits are made and the original work is properly cited (including links to both the formal publication through the relevant DOI and the license). See: https://creativecommons.org/licenses/by-nc-nd/4.0/.

\section{References}

1. Vuitton D. Alveolar echinococcosis of the liver: a parasitic disease in search of a treatment. Hepatology 1990;12:617-8.

2. Wen H, Vuitton L, Tuxun T, et al. Echinococcosis: Advances in the 21st Century. Clin Microbiol Rev 2019;32.

3. Craig P. Echinococcus multilocularis. Curr Opin Infect Dis 2003;16:437-44.

4. Qiu Y, Yang X, Shen S, et al. Vascular infiltrationbased surgical planning in treating end-stage hepatic alveolar echinococcosis with ex vivo liver resection and autotransplantation. Surgery 2019;165:889-96.

5. Yang X, Qiu Y, Huang B, et al. Novel techniques and preliminary results of ex vivo liver resection and autotransplantation for end-stage hepatic alveolar
Wang et al. A new sarcopenia score prognostic for PCs for HAE

echinococcosis: A study of 31 cases. Am J Transplant 2018;18:1668-79.

6. Shen S, Qiu Y, Yang X, et al. Remnant Liver-to-Standard Liver Volume Ratio Below 40\% is Safe in Ex Vivo Liver Resection and Autotransplantation. J Gastrointest Surg 2019;23:1964-72.

7. Aji T, Dong JH, Shao YM, et al. Ex vivo liver resection and autotransplantation as alternative to allotransplantation for end-stage hepatic alveolar echinococcosis. J Hepatol 2018;69:1037-46.

8. Lobo DN, Gianotti L, Adiamah A, et al. Perioperative nutrition: Recommendations from the ESPEN expert group. Clin Nutr 2020;39:3211-27.

9. Probst P, Fuchs J, Schön MR, et al. Prospective study to evaluate the prognostic value of different nutritional assessment scores in liver surgery: NURIMAS Liver (DRKS00006340). Hepatobiliary Surg Nutr 2020;9:400-13.

10. Cruz-Jentoft AJ, Bahat G, Bauer J, et al. Sarcopenia: revised European consensus on definition and diagnosis. Age Ageing 2019;48:16-31.

11. Czigany Z, Kramp W, Bednarsch J, et al. Myosteatosis to predict inferior perioperative outcome in patients undergoing orthotopic liver transplantation. Am J Transplant 2020;20:493-503.

12. Ooi PH, Hager A, Mazurak VC, et al. Sarcopenia in Chronic Liver Disease: Impact on Outcomes. Liver Transpl 2019;25:1422-38.

13. Simonsen C, de Heer P, Bjerre ED, et al. Sarcopenia and Postoperative Complication Risk in Gastrointestinal Surgical Oncology: A Meta-analysis. Ann Surg 2018;268:58-69.

14. Joglekar S, Asghar A, Mott SL, et al. Sarcopenia is an independent predictor of complications following pancreatectomy for adenocarcinoma. J Surg Oncol 2015;111:771-5.

15. Nakamura R, Inage $Y$, Tobita R, et al. Sarcopenia in Resected NSCLC: Effect on Postoperative Outcomes. J Thorac Oncol 2018;13:895-903.

16. Englesbe MJ, Patel SP, He K, et al. Sarcopenia and mortality after liver transplantation. J Am Coll Surg 2010;211:271-8.

17. Okumura S, Kaido T, Hamaguchi $Y$, et al. Impact of Skeletal Muscle Mass, Muscle Quality, and Visceral Adiposity on Outcomes Following Resection of Intrahepatic Cholangiocarcinoma. Ann Surg Oncol 2017;24:1037-45.

18. Fujiwara N, Nakagawa H, Kudo Y, et al. Sarcopenia, intramuscular fat deposition, and visceral adiposity 
independently predict the outcomes of hepatocellular carcinoma. J Hepatol 2015;63:131-40.

19. Lin ZH, Xin YN, Dong QJ, et al. Performance of the aspartate aminotransferase-to-platelet ratio index for the staging of hepatitis C-related fibrosis: an updated metaanalysis. Hepatology 2011;53:726-36.

20. Ke M, Xu T, Li N, et al. Prognostic nutritional index predicts short-term outcomes after liver resection for hepatocellular carcinoma within the Milan criteria. Oncotarget 2016;7:81611-20.

21. Johnson PJ, Berhane S, Kagebayashi C, et al. Assessment of liver function in patients with hepatocellular carcinoma: a new evidence-based approach-the ALBI grade. J Clin Oncol 2015;33:550-8.

22. Li L, Liu C, Yang J, et al. Early postoperative controlling nutritional status (CONUT) score is associated with complication III-V after hepatectomy in hepatocellular carcinoma: A retrospective cohort study of 1,334 patients. Sci Rep 2018;8:13406.

23. Tamai Y, Iwasa M, Kawasaki Y, et al. Ratio between estimated glomerular filtration rates of creatinine and cystatin C predicts overall survival in patients with hepatocellular carcinoma. Hepatol Res 2019;49:153-63.

24. Joliat GR, Labgaa I, Demartines N, et al. Preoperative albumin level is a marker of alveolar echinococcosis recurrence after hepatectomy. World J Gastroenterol 2017;23:853-8.

25. Labgaa I, Joliat GR, Demartines N, et al. Serum albumin is an early predictor of complications after liver surgery.

Dig Liver Dis 2016;48:559-61.

26. Harimoto N, Yoshizumi T, Inokuchi S, et al. Prognostic Significance of Preoperative Controlling Nutritional Status (CONUT) Score in Patients Undergoing Hepatic Resection for Hepatocellular Carcinoma: A Multiinstitutional Study. Ann Surg Oncol 2018;25:3316-23.

27. Guidelines for treatment of cystic and alveolar echinococcosis in humans. WHO Informal Working Group on Echinococcosis. Bull World Health Organ 1996;74:231-42.

28. Onodera T, Goseki N, Kosaki G. [Prognostic nutritional index in gastrointestinal surgery of malnourished cancer patients]. Nihon Geka Gakkai Zasshi 1984;85:1001-5.

29. Suh B, Park S, Shin DW, et al. Low albumin-to-globulin ratio associated with cancer incidence and mortality in generally healthy adults. Ann Oncol 2014;25:2260-6.

30. Yang X, Qiu Y, Wang W, et al. Risk factors and a simple model for predicting bile leakage after radical hepatectomy in patients with hepatic alveolar echinococcosis. Medicine
(Baltimore) 2017;96:e8774.

31. Rahbari NN, Garden OJ, Padbury R, et al.

Posthepatectomy liver failure: a definition and grading by the International Study Group of Liver Surgery (ISGLS). Surgery 2011;149:713-24.

32. Kern P, Wen H, Sato N, et al. WHO classification of alveolar echinococcosis: principles and application. Parasitol Int 2006;55 Suppl:S283-7.

33. Dindo D, Demartines N, Clavien PA. Classification of surgical complications: a new proposal with evaluation in a cohort of 6336 patients and results of a survey. Ann Surg 2004;240:205-13.

34. Mourtzakis M, Prado CM, Lieffers JR, et al. A practical and precise approach to quantification of body composition in cancer patients using computed tomography images acquired during routine care. Appl Physiol Nutr Metab 2008;33:997-1006.

35. Iasonos A, Schrag D, Raj GV, et al. How to build and interpret a nomogram for cancer prognosis. J Clin Oncol 2008;26:1364-70.

36. Yang C, He J, Yang X, et al. Surgical approaches for definitive treatment of hepatic alveolar echinococcosis: results of a survey in 178 patients. Parasitology 2019;146:1414-20.

37. Khuri SF, Henderson WG, DePalma RG, et al. Determinants of long-term survival after major surgery and the adverse effect of postoperative complications. Ann Surg 2005;242:326-41; discussion 341-3.

38. Lou N, Chi CH, Chen XD, et al. Sarcopenia in overweight and obese patients is a predictive factor for postoperative complication in gastric cancer: A prospective study. Eur J Surg Oncol 2017;43:188-95.

39. Kobayashi A, Kaido T, Hamaguchi Y, et al. Impact of Sarcopenic Obesity on Outcomes in Patients Undergoing Hepatectomy for Hepatocellular Carcinoma. Ann Surg 2019;269:924-31.

40. Hamaguchi Y, Kaido T, Okumura S, et al. Muscle Steatosis is an Independent Predictor of Postoperative Complications in Patients with Hepatocellular Carcinoma. World J Surg 2016;40:1959-68.

41. Harimoto N, Hoshino H, Muranushi R, et al. Skeletal Muscle Volume and Intramuscular Adipose Tissue Are Prognostic Predictors of Postoperative Complications After Hepatic Resection. Anticancer Res 2018;38:4933-9.

42. Kuroki LM, Mangano M, Allsworth JE, et al. Preoperative assessment of muscle mass to predict surgical complications and prognosis in patients with endometrial cancer. Ann Surg Oncol 2015;22:972-9. 


\section{Page 18 of 18}

43. Ali S, Garcia JM. Sarcopenia, cachexia and aging: diagnosis, mechanisms and therapeutic options - a minireview. Gerontology 2014;60:294-305.

44. Sandri M. Signaling in muscle atrophy and hypertrophy. Physiology (Bethesda) 2008;23:160-70.

45. Drummond MJ, Dreyer HC, Fry CS, et al. Nutritional and contractile regulation of human skeletal muscle protein synthesis and mTORC1 signaling. J Appl Physiol (1985) 2009;106:1374-84.

46. Prado CM, Lieffers JR, McCargar LJ, et al. Prevalence and clinical implications of sarcopenic obesity in patients with solid tumours of the respiratory and gastrointestinal tracts: a population-based study. Lancet Oncol 2008;9:629-35.

47. Awad S, Constantin-Teodosiu D, Macdonald IA, et al. Short-term starvation and mitochondrial dysfunction a possible mechanism leading to postoperative insulin resistance. Clin Nutr 2009;28:497-509.

48. Tilg H, Moschen AR. Adipocytokines: mediators linking adipose tissue, inflammation and immunity. Nat Rev Immunol 2006;6:772-83.

49. Brons C, Grunnet LG. MECHANISMS IN ENDOCRINOLOGY: Skeletal muscle lipotoxicity in insulin resistance and type 2 diabetes: a causal mechanism or an innocent bystander? Eur J Endocrinol

Cite this article as: Wang $\mathrm{T}$, Yang $\mathrm{X}$, Wang $\mathrm{W}$, Chen $\mathrm{T}$, Kong J, Shen S, Chen Y, Wei G, Yu D, Wang C, Li M, Rui S, Luo B, Wang W. A new sarcopenia score prognostic for postoperative complications in hepatic alveolar echinococcosis: a multicenter retrospective study. Ann Transl Med 2020;8(21):1398. doi: 10.21037/atm-20-1960a
Wang et al. A new sarcopenia score prognostic for PCs for HAE

2017;176:R67-R78.

50. Bhanji RA, Montano-Loza AJ, Watt KD. Sarcopenia in Cirrhosis: Looking Beyond the Skeletal Muscle Loss to See the Systemic Disease. Hepatology 2019;70:2193-203.

51. Shariq OA, Hanson KT, McKenna NP, et al. Does Metabolic Syndrome Increase the Risk of Postoperative Complications in Patients Undergoing Colorectal Cancer Surgery? Dis Colon Rectum 2019;62:849-58.

52. Lee IJ, Seong J, Bae JI, et al. Effect of Oral Supplementation with Branched-chain Amino Acid (BCAA) during Radiotherapy in Patients with Hepatocellular Carcinoma: A Double-Blind Randomized Study. Cancer Res Treat 2011;43:24-31.

53. Kayaalp C, Bzeizi K, Demirbag AE, et al. Biliary complications after hydatid liver surgery: incidence and risk factors. J Gastrointest Surg 2002;6:706-12.

54. Demircan O, Baymus M, Seydaoglu G, et al. Occult cystobiliary communication presenting as postoperative biliary leakage after hydatid liver surgery: are there significant preoperative clinical predictors? Can J Surg 2006;49:177-84.

55. Lee DH, Blomhoff R, Jacobs DR, Jr. Is serum gamma glutamyltransferase a marker of oxidative stress? Free Radic Res 2004;38:535-9. 